Recepción: 20 / 04 / 2017

Aceptación: 20 / 05 / 2017

Publicación: 15 / 07 / 2017

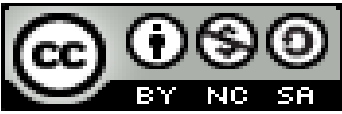

Ciencias Médicas

Artículo Científico

\title{
Prevalencia de infecciones vaginales en adolescentes
}

\author{
Prevalence of vaginal infections in adolescents
}

\section{Prevalência de infecções vaginais em adolescentes}

Ecuador E. Montenegro Moran ${ }^{\mathrm{I}}$ ecuador.montenegrom@ug.edu.ec

Julio De la Torre Chávez ${ }^{\text {II }}$ julio.delatorrec@ug.edu.ec

Kathiusca Hernández-Velásquez ${ }^{\text {III }}$ kathiusca.hernandezv@ug.edu.ec

Marlene Saltos-Calvache IV marle.saltosc@ug.edu.ec

Correspondencia: ecuador.montenegrom@ug.edu.ec

Médico, Universidad de Guayaquil, Guayaquil, Ecuador. Médico, Universidad de Guayaquil, Guayaquil, Ecuador. Médico, Universidad de Guayaquil, Guayaquil, Ecuador. Médico, Universidad de Guayaquil, Guayaquil, Ecuador. 


\section{Resumen.}

El problema las infecciones vaginales representa el segundo problema que presentan las adolescentes en edad reproductiva, es una causa frecuente de consulta ginecológica en nuestro medio. Los objetivos de la investigación fueron, determinar la prevalencia de las infecciones vaginales en las adolescentes de los colegios Eloy Alfaro Duran y Provincia del Cañar. La población a investigar fueron 250 adolescentes que recibieron las charlas sobre infecciones vaginales de las cuáles voluntariamente 106 adolescentes contestaron la encuesta y la muestra fue escogida del $100 \%$ de las encuestas realizadas las adolescentes, y quedando conformada por 106 alumnas, además se aplicó los criterios de inclusión y exclusión para que la muestra sea lo más representativa de la realidad. Para la recolección de la muestra se aplicó como instrumento de investigación la encuesta estructuradas con preguntas cerradas. Los resultados fueron, de los 106 adolescentes, se obtuvo que 77 presentaron infecciones vaginales que representa un 73\%, lo que representa una altísima incidencia de adolescentes que presentaron infecciones vaginales, los resultados son concluyente como, el grupo etario más frecuente es entre las edades de 15 a 17 años con un $38.1 \%$, los signos y síntomas más predominantes fueron la vaginosis bacteriana con el 58\%, en segundo lugar la tricomoniasis con el $30 \%$. Y por último la candidiasis con el 12\%, por esta razón nuestra propuesta fue estructurada para indagar el porcentaje de adolescentes portadoras de infección vaginal, lo que llevará a obtener datos reales que servirán como ayuda para poder concientizar a las adolescentes, y establecer un protocolo de capacitación y prevención de infecciones vaginales en las adolescentes de instituciones educativas encaminada a prevenir las infecciones vaginales.

Palabras Clave: Infecciones vaginales; adolescentes; vaginosis bacteriana. 


\section{Abstract.}

The problem of vaginal infections represents the second problem presented by adolescents of reproductive age, is a frequent cause of gynecological consultation in our country. The objectives of the investigation were to determine the prevalence of vaginal infections in the adolescents of the schools "Eloy Alfaro Duran" and "Provincia del Cañar". The population to be investigated were 250 adolescents who received the talks about vaginal infections, of which voluntarily 106 adolescents answered the survey and the sample was chosen from $100 \%$ of the surveys carried out for the adolescents, and was made up of 106 students, in addition the criteria were applied Of inclusion and exclusion so that the sample is the most representative of reality. For the collection of the sample was applied as a research instrument the survey structured with closed questions. The results were, of the 106 adolescents, it was found that 77 had vaginal infections representing 73\%, which represents a very high incidence of adolescents who had vaginal infections, the results are conclusive as, the most frequent age group is between the ages From 15 to 17 years old with $38.1 \%$, the most prevalent signs and symptoms were bacterial vaginosis with $58 \%$, secondly trichomoniasis with $30 \%$. And lastly the candidiasis with $12 \%$, for this reason our proposal was structured to investigate the percentage of adolescents carrying vaginal infection, which will lead to obtain real data that will serve as an aid to raise awareness among adolescents, and establish a protocol Training and prevention of vaginal infections in adolescents of educational institutions aimed at preventing vaginal infections.

Keywords: Vaginal infections; teenagers; Bacterial vaginosis. 


\section{Resumo.}

O problema das infecções vaginais representa o segundo problema apresentado por adolescentes em idade reprodutiva, é uma causa frequente de consultas ginecológicas em nosso país. Os objetivos da investigação foram determinar a prevalência de infecções vaginais nos adolescentes das escolas "Eloy Alfaro Duran" e "Provincia del Cañar". A população a ser investigada foi de 250 adolescentes que receberam as conversas sobre infecções vaginais, das quais 106 adolescentes voluntariamente responderam a pesquisa e a amostra foi escolhida em $100 \%$ das pesquisas realizadas para adolescentes e foi composta por 106 alunos, em Além disso, os critérios foram aplicados de inclusão e exclusão para que a amostra seja a mais representativa da realidade. Para a coleta da amostra foi aplicado como um instrumento de pesquisa a pesquisa estruturada com perguntas fechadas. Os resultados foram dos 106 adolescentes, descobriu-se que 77 tinham infecções vaginais representando 73\%, o que representa uma incidência muito alta de adolescentes que tiveram infecções vaginais, os resultados são conclusivos, a faixa etária mais freqüente é entre as idades. De De 15 a 17 anos com $38,1 \%$, os sinais e sintomas mais prevalentes foram vaginose bacteriana com $58 \%$, em segundo lugar, tricomoníase com $30 \%$. E, por último, a candidíase com $12 \%$, por esta razão, nossa proposta foi estruturada para investigar a porcentagem de adolescentes portadores de infecção vaginal, o que levará à obtenção de dados reais que servirão de ajuda para aumentar a conscientização dos adolescentes e estabelecer um protocolo Treinamento e Prevenção de infecções vaginais em adolescentes de instituições educacionais voltadas para a prevenção de infecções vaginais.

Palavras chave: Infecções vaginais; adolescentes; a vaginose bacteriana. 


\section{Introducción.}

En la actualidad hay un alto porcentaje de adolescentes que acuden a la emergencia de los centro de Salud por amenaza de partos pretérmino, amenazas de aborto, abortos espontaneos, ruptura prematura de membranas, coriamnionitis por causa de infecciones vaginales. $(1,2)$

Las patologías vaginales son causas muy frecuentes de consulta médica general, pueden ser infecciosas y no infecciosas. Las causas infecciosas de flujo vaginal abarcan desde virus hasta los protozoos pasando por las bacterias y los hongos. $(3,4)$

El ecosistema vaginal normal es reconocido como un importante mecanismo de defensa del huésped contra dichas infecciones, ya sea por la exclusión competitiva de los microor-ganismos, la producción de ácido láctico por los lactobacillus presentes, o bien por la producción de bacteriocinas y/o peróxido de hidrógeno. (5)

Sin embargo, también se reconocen una serie de factores que pueden alterar el citado ecosistema, como los relacionados con la edad, inicio temprano de relaciones sexuales, embarazo, higiene personal, número de compañeros sexuales, métodos anticonceptivos, etc. Evidentemente, mientras más se conozca sobre los factores del huésped que condicionan la aparición de estas infecciones y se incida en ellos, su frecuencia y complicaciones tenderán a disminuir. (6)

Normalmente existe alguna cantidad de flujo vaginal, especialmente en mujeres en edad de procrear. Un flujo vaginal que súbitamente cambia de color, olor o consistencia, aumenta o disminuye significativamente en cantidad, puede ser indicio de un problema subyacente, como una infección. 
La vaginosis bacteriana es una alteración de la flora vaginal bacteriana normal donde hay una reducción de los lactobacillus productores de peróxido de hidrógeno y un incremento de prevalencia y concentración de Gardnerella vaginalis, Mycoplasma hominis y Anaero-bios: Mobiluncus spp, Prevotella spp, Bacteroides spp y Peptoestreptococo.

La Vaginitis es cualquier tipo de inflamación o infección de la vagina, es un problema ginecológico común que afecta a mujeres de todas las edades. Puede presentarse cuando las paredes de la vagina se inflaman debido a un agente irritante que trastorna el equilibrio de la región vaginal; puede ser provocada por diversos factores como: bacterias, hongos, virus, elementos químicos y algunas veces también puede presentarse por la trasmisión de microorganismos de una pareja sexual a otra.

La infección por levaduras ocurre cuando ciertos factores internos o externos alteran el ambiente natural de la vagina y propician el crecimiento anormal de hongos microscópi-cos, entre los cuales el más común es Cándida albicans.

La infección por protozoarios es causada, generalmente, por Trichomona vaginalis, suele trasmitirse por contacto sexual.

La evaluación de las Infecciones Vaginales requiere un interrogatorio clinicamente detallado y exahustivo, examen físico y análisis de la secreción vaginal de la paciente, este último cumple un papel importante en el laboratorio microbiológico ya que permite la identificación de microorganismos asociados a una infección vaginal, mediante técnicas adecuadas, y con controles de calidad para obtener resultados veraces. 
La presente investigación tiene como objetivo determinar la prevalencia de infecciones vaginales en adolescentes de los colegios Eloy Alfaro Duran y provincia del Cañar durante el periodo septiembre del 2016 a enero 2017.

\section{Materiales y métodos.}

La Investigación se la realizo en los colegios Eloy Alfaro Durán y Provincia del Cañar ubicados en el Cantón Durán. En el canton Duran tiene 240000 habitantes en la cual se ha visto afectada a la juventud que están en los colegios tiene un gran porcentaje de adolescentes madres de familia, las misma que por su desconocimiento una vez empezada su vida sexual no se cuidan y son las más vulnerables de adquirir infecciones vaginales. Es por eso este estudio que de impartirá en los principales colegios de Duran. Con el fin de prevenir a nuestra juventud adolescente.

\section{La muestra}

Total de 106 encuestas realizadas a las adolescentes de los colegios Eloy Alfaro Duran y provincia del Cañar.

En esta etapa se utilizó un modelo de tabulación para la recolección de datos en cuya información se encuentra la edad de las adolescentes, inicio de relaciones sexuales, infecciones vaginales presuntivas, si recibieron tratamiento médico.

Para la correcta interpretación de los datos se utilizó el análisis estadístico del programa Microsoft Excel 9.0. La elaboración de tablas, resumen con gráficos explicativos de la información. 
Ecuador E. Montenegro Moran; Julio De la Torre Chávez; Kathiusca Hernández-Velásquez; Marlene Saltos-Calvache

\section{Resultados.}

Tabla 1.- Adolescentes que tienen Relaciones Sexuales.

\begin{tabular}{|cc|}
\hline Relaciones Sexuales & Adolecentes \\
\hline si & 77 \\
\hline no & 29 \\
\hline Total & $\mathbf{1 0 6}$ \\
\hline
\end{tabular}

De 106 adolescentes estudiantes se los colegios Eloy Alfaro Dur\{an procincia del Cañar el 73\% son sexualmente activas.

Tabla 2.- Adolescentes Sexualmente activas por edades.

\begin{tabular}{|ccc|}
\hline Edad & Adolecentes & $\%$ \\
\hline 15 & 8 & $17 \%$ \\
\hline 16 & 13 & $25 \%$ \\
\hline 17 & 19 & $48 \%$ \\
\hline 18 & 37 & $10 \%$ \\
\hline Total & $\mathbf{7 7}$ & \\
\hline
\end{tabular}

Se clasifica por edades el porcentaje de adolescentes que tienen relaciones sexuales. 
Tabla 3.- Inicio de Actividad Sexual de las Adolescentes

\begin{tabular}{|ccc|}
\hline Edad & Adolecentes & $\%$ \\
\hline 11 & 1 & $1.3 \%$ \\
\hline 12 & 14 & $18.2 \%$ \\
\hline 13 & 22 & $28.6 \%$ \\
\hline 14 & 19 & $24.7 \%$ \\
\hline 15 & 15 & $19.5 \%$ \\
\hline 16 & 6 & $7.8 \%$ \\
\hline
\end{tabular}

Total 77

Este cuadro ase analiza la edad en que las adolescentes empiezan su actividad sexual, dando como el mayor porcentaje de las adolescentes empiezan a tener relaciones sexuales a los 13 años. Este dato es muy importante ya que queremos enfocarnos a que adolescentes vamos a a capacitar de acuerdo a la necesidad.

Tabla 4.- Adolescentes con Sintomatología de Infecciones Vaginales.

\begin{tabular}{|cc|}
\hline Edad & Adolecentes \\
\hline 15 & 10 \\
\hline 16 & 15 \\
\hline 17 & 35 \\
\hline 18 & 4 \\
\hline
\end{tabular}

Total

64

Es análisis nos da la pauta para determina el número de adolescentes que padecen infecciones vaginales. 
Tabla 5.- Diagnostico presuntico de infecciones vaginales.

\begin{tabular}{|cc|}
\hline Diagnostico Presuntivo & Adolecentes \\
\hline Candidiasis & 8 \\
\hline Tricomoniasis & 19 \\
\hline Vaginosis Bacteriana & 37 \\
\hline Total & $\mathbf{6 4}$ \\
\hline
\end{tabular}

De acuerdo a las características de la sintomatología de las adolescentes se puede llegar aún diagnostico presuntivo del tipo de infecciones vaginales que padecen las adolescentes.

Tabla 6.- Diagnostico Presuntivo - Candidiasis.

\begin{tabular}{|cc|}
\hline Edad & Adolecentes \\
\hline 15 & 1 \\
\hline 16 & 2 \\
\hline 17 & 4 \\
\hline 18 & 1 \\
\hline
\end{tabular}

Según la sintomatología que presentan las adolescentes se puede decir que el $12 \%$ de adolescentes padecen o padecieron Candidiasis vaginal.

Tabla 7.- Adolecentes que acuden a la consulta medica

\begin{tabular}{|cc|}
\hline Consulta medica & Adolecentes \\
\hline no & 3 \\
\hline si & 5 \\
\hline
\end{tabular}

Podemos decir que de cada 8 adolescentes que presentan sintomatología para candidiasis vaginal solo el $63 \%$ acuden a la consulta médica. Y el $37 \%$ son las posible pacientes que presentaran en un futuro no muy lejano complicaciones ginecológicas. 
Tabla 8.- Adolecentes que siguieron Tratamiento

\begin{tabular}{|cc|}
\hline Tratamiento & Adolecentes \\
\hline automedicación & 3 \\
\hline $\mathrm{Si}$ & 5 \\
\hline
\end{tabular}

Este análisis nos dice que el $63 \%$ de las pacientes que padecen de candidiasis acuden a la consulta médica y que el $37 \%$ se automedico y este $37 \%$ es en sonde va a reincidir la infección vaginal.

Tabla 9.- De acuerdo a la sintomatología expresada en la encuesta las adolescentes que presentaron tricomoniasis.

\begin{tabular}{|cc|}
\hline Edad & Adolecentes \\
\hline 15 & 3 \\
\hline 16 & 6 \\
\hline 17 & 9 \\
\hline 18 & 1 \\
\hline
\end{tabular}

Según los resultados de las encuestas del $73 \%$ de las adolescentes que son sexualmente activas, el $30 \%$ de las adolescentes padecieron tricomoniasis.

Tabla 10.- Adolecentes que acudieron a Consulta Médica - Tricomoniasis

\begin{tabular}{|cc|}
\hline Consulta medica & Adolecentes \\
\hline no & 5 \\
\hline si & 14 \\
\hline
\end{tabular}


Las adolescentes que padecieron tricomoniasis solo el $74 \%$ acudieron a al consulta médica y recibieron tratamiento. El 26\% que padecieron tricomoniais no acudió a la consulta médica.

\section{Tabla 11.- Adolecentes que siguieron Tratamiento}

\begin{tabular}{|cc|}
\hline Tratamiento & Adolecentes \\
\hline automedicación & 5 \\
\hline si & 14 \\
\hline
\end{tabular}

El 26\% de adolescentes que padecieron tricomoniasis se automedicaron aumentando el riesgo.

\section{Tabla 12.- Adolecentes con Diagnostivo Presuntivo de Vaginosis Bacteriana}

\begin{tabular}{|cc|}
\hline Edad & Adolecentes \\
\hline 15 & 6 \\
\hline 16 & 7 \\
\hline 17 & 22 \\
\hline 18 & 2 \\
\hline
\end{tabular}

Este cuadro estadístico nos indica que la vaginosis bacteriana es la infección vaginal más frecuente en las adolescentes que son sexualmente activas.

Tabla 13.- Adolecentes que acuedieron a Consulta Médica - Vaginosis Bacteriana

\begin{tabular}{|cc|}
\hline Consulta medica & Adolecentes \\
\hline no & 6 \\
\hline si & 31 \\
\hline
\end{tabular}

De las adolescentes que padecieron vaginosis bacteriana el $84 \%$ acudió a la consulta médica. 


\section{Tabla 14.- Adolecentes que siguieron Tratamiento - Vaginosis Bacteriana}

\begin{tabular}{|cc|}
\hline Tratamiento & Adolecentes \\
\hline automedicacion & 6 \\
\hline si & 31 \\
\hline
\end{tabular}

Las que recibieron tratamiento médico y las que se auto medicaron.

\section{Conclusiones.}

De las ambas instituciones el total fue 106 adolescentes de las cuales 77 eran sexualmente activas y 64 presentaron infecciones vaginales que representa $60.33 \%$ en relación a un $39.67 \%$ que no tuvo infección.

Las infecciones vaginales más frecuentes fueron $58 \%$ vaginosis bacteriana, 30\% tricomoniasis y $12 \%$ candidiasis.

El inicio de relaciones sexuales a temprana edad influye en el incremento de infecciones vaginales precoz en las adolescentes de los colegios Eloy Alfaro Durán y Provincia del Cañar.

\section{Recomendaciones.}

Que se impartan Seminario y charlas a las adolescentes de las instituciones educativas para la prevención de la infecciones Vaginales, de esta manera concienciar a las adolescentes al no iniciar sus relaciones sexuales a temprana edad.

Promover trabajos de investigación relacionados con él tema, incentivando a las adolescentes a que una vez iniciada su vida sexual, acudan al control ginecológico. 
Estudiar otros factores de riesgo como nivel socioeconómico, conductas sexuales que puedan estar relacionados con el incremento de infecciones vaginales en las adolescentes.

Educar no solo a los estudiantes secundarios adolescentes sino también a sus familias para dialogar el tema de sexualidad dentro del hogar sin tabú o complejos, para que los padres y tutores puedan orientar correctamente desde niños.

\section{Bibliografía.}

1. Ya w, Reifer C, Miller L. Efficacy of vaginal probiotic capsules for recurrent bacterial vaginosis: a doubleblind, randomized, placebo-controlled study. Am J Obstet Gynecol. Am J Obstet Gynecol ago; 203(2): p. $120-126$.

2. Klatt T, Cole D, Eastwood D. Factors associated with recurrent bacterial vaginosis. J Reprod Med. 2010 ene; 55(1-2): p. 55-61.

3. Loh K, Sivalingam N. Recurrent vaginal candidiasis. Med J Malaysia. 2003 dec; 58(5).

4. Ehrström S, Daroczy K, Rylander E, Samuelsson C, Johannesson U. Lactic acid bacteria colonization and clinical outcome after probiotic supplementation in conventionally treated bacterial vaginosis and vulvovaginal candidiasis. Microbes Infect. 2010 sep; 12(10).

5. De Seta F, Parazzini F, De Leo R, Banco R, Maso G. Lactobacillus plantarum P17630 for preventing Candida vaginitis recurrence: a retrospective comparative study. Eur J Obstet Gynecol Reprod Biol. 2014 nov; 182(183).

6. Vázquez F, García MJ. Trichomonas vaginalis: tratamiento y resistencia a nitroimidazoles. Enfermedades Infecciosas y microbiología clínica. 2001; 19(1). 